\title{
Regenerative Surgery Unit (RSU) a new model of care
}

\author{
Valerio Di Nicola ${ }^{1,2 *}$ \\ ${ }^{1}$ West Sussex Hospitals NHS Foundation Trust- Worthing Hospital, BN112DH, UK \\ ${ }^{2}$ Regenerative Surgery Unit, Villa Aurora Hospital, Foligno, Italy
}

\begin{abstract}
Regenerative Surgery $(R S)$ defines all the Regenerative Medicine $(R M)$ treatments that can be applied through minimally invasive surgical procedures. This biomedical approach includes the use of stem cells, various local biochemical mediators, and tissue engineering.

The Regenerative Surgery Unit (RSU) is a new model of health care governance integrating clinical, scientific and research activities.

$R S U$ works in a multidisciplinary setting involving surgeon, biologist, immunologist, laboratory technician, radiologist, and physiotherapist.

$R S U$ setting also aims to generate a special relation between doctor and patient. The aim of this relationship is to establish a solid and mutually beneficial alliance, founded on medical ethics, between doctor and patient.

$R S U$ organises clinical services through ambulatory care and day surgery settings. The scientific and research activities are integrated as components of daily medical practice.

This article describes some established procedures in $R S$ and some refinements that are more recently available as a result of our clinical and research experience in $R M$ fields.

Health Tourism is one element of the sustainable growth model we are developing in the RSU-Villa Aurora Hospital, Foligno (Italy).
\end{abstract}

\section{Introduction}

Regenerative Medicine (RM) is a branch of translational research involving tissue engineering and molecular biology. $R M$ deals with the process of regenerating and replacing dysfunctional cells, tissues, and organs; the aim is to restore normal healthy function.

$R M$ intervenes on injured tissues and organs by stimulating body self-repair mechanisms to recover structure and functionality. Biomedical approaches within the field of $R M$ may involve the use of stem cells, biologically active molecules, and tissue engineering.

Regenerative Surgery (RS) defines all the $R M$ treatments that can be clinically applied through minimally invasive surgical procedures. $R S$ opens new possibilities for the delivery of ethical and effective care. Realizing the full benefits requires an appropriate clinical setting and management approach. Patient understanding and safety together with clinical understanding of the need for and benefits of continuous improvement are the cornerstones of a robust and positive alliance with patients. This article reports the model we are applying to Villa Aurora Hospital-Regenerative Surgery Unit- Foligno (Italy).

\section{Regenerative Surgery Unit (RSU) model and organization}

The RSU model integrates clinical, scientific and research activities to create valuable assets. The clinical asset is structured to deliver multidisciplinary and minimally invasive procedures with the aim to halt and reverse symptoms and where possible the underlying conditions. This is achieved through tissue recovery and regeneration. The scientific asset includes the constant collection, updating and communication of data, observations, and findings. It also includes education and training, publications, meetings and conferences and development of scientific associations. The research asset encompasses in vitro investigations, experimental models, and clinical trials. $R S U$ works in a multidisciplinary team involving surgeon, biologist, immunologist, laboratory technician, radiologist, and physiotherapist.

RSU clinical activity is centered on ambulatory care or day surgery procedures.

Patients who receive regenerative treatments can 'walk in and out' of $R S U$ within few hours. Dedicated facilities allow $R S$ specialists to deliver safe minimally invasive procedures.

Areas of intervention in our Regenerative Surgery Unit:

\section{Orthopaedic $R S$}

- Early Osteoarthritis (OA)

- Advanced OA

- Traumatic and degenerative damage to tendons and ligaments.

${ }^{*}$ Correspondence to: Valerio Di Nicola, West Sussex Hospitals NHS Foundation Trust- Worthing Hospital BN112DH, UK, Tel: +447557904032; E-mail: dinicolavalerio@gmail.com

Key words: surgery settings, health care governance, RM fields, medical practice Received: May 04, 2020; Accepted: May 22, 2020; Published: May 25, 2020 


\section{Plastic RS}

- diabetic foot ulcers (DFU)

- pressure ulcers (PU)

- venous leg ulcers (VLUs).

\section{General $R S$}

- PRF- hernia repair

- perianal fistulas

- preliminary experience in faecal incontinence

\section{Aesthetic RS}

- filling skin defects

- wrinkles

- stimulation for hair regrowth and hair transplantation.

\section{Maxillofacial $R S$}

- periodontal bony defects

- PRF- implant surgery

\section{RS procedures}

These treatments have the common aim of improving symptoms and restoring homeostasis to local cells and tissue regeneration.

\section{Materials employed}

Platelet-rich plasma (PRP) is a concentrate of platelet-rich plasma proteins derived from centrifuged whole blood.

$P R P$ variably contains several growth factors including Platelet derived growth factor $(P D G F)$; Vascular endothelial growth factor (VEGF); Transforming growth factor beta-1 (TGF-b1); Epidermal growth factor $(E G F)$; Basic fibroblast growth factor $(F G F-\beta)$ and Insulin-like growth factor-1 (IGF-1) [1].

Preparations have been categorized by content into four main groups: $P P R P$ (pure PRP), $L-P R P$ (leukocyte and PRP), $P-P R F$ (pure platelet-rich fibrin) and, $L-P R F$ (leukocyte and platelet-rich fibrin) [1].

Literature shows that $P R P$ can stimulate an antiinflammatory, proliferative and remodelling response inducing mesenchymal tissue regeneration [2].

Specifically, in osteoarthritis $(O A)$, chondrogenic differentiating properties were found in vitro as well as in vivo in mice and rabbits, indicating a major role in regeneration of cartilage [3-7].

These PRP biological properties are increasingly used for $O A$ treatments, maxillofacial and dental procedures, plastic surgery and aesthetic treatments and their use has recently been expanding in general surgery $[8,9]$.

The final goal is the stimulation of resident MSCs to promote a regenerative microenvironment.

MSCs stimulate local inflammatory-immune modulation and trophic effects and ultimately facilitate tissue regeneration [10-14].

\section{MSCs Therapy}

In 2001 the FDA issued a set of regulations governing cellular and tissue-based therapies. The basic principle of these guidelines was that cellular and tissue therapies that require minimal to no manipulation of autologous tissue do not required FDA approval [15].

MSCs isolated from different sources such as bone marrow, adipose tissue, cord blood, amniotic fluid, omentum, peripheral blood etc, showed in vitro capacity to differentiate in all mesenchymal lineages: fibroblasts, chondroblasts, adipocytes, muscle-cells, osteoblasts and other mesenchymal lineages [14-17].

Autologous ASCs offer advantages from regulatory, histocompatibility, and infectious perspectives.

Autologous MSCs are the most frequently used in clinical practice.

For autologous MSCs treatments in $O A$ and plastic-aesthetic surgery the use of adipose tissue-derived stem cells (ASCs) is the most common and the most studied. The use of peripheral blood stem cells (PBSCs) is becoming popular [2].

Modern therapeutic strategies rely on the combination of MSCs and tissue engineering so that MSCs suspensions combined with biodegradable materials may improve cell survival and tissue regeneration capacity [18]. As with other MSCs it has been supposed that ASCs delivered into injured tissue induce repair and regeneration via paracrine effects. The ASCs stimulate the resident stem cells and promote their differentiation along the required lineage pathway. Furthermore, ASCs similar to BM-MSCs can deliver new mitochondria to damaged cells, thereby rescuing aerobic metabolism $[10,11,12,19]$.

Therefore, against the initial assumption that MSCs might in vivo reproduce the in vitro findings, evidence increasingly suggests that the main therapeutic function of MSCs could be the paracrine effect to induce a local regenerative microenvironment $[10,20]$.

In degenerative $O A, A S C s$ can be culture-expanded and then injected into the joint or they can be harvested and directly injected as stromal vascular fraction SVF [14,21,22]. Further, ASCs have been used as mixed injections in combination with GFs, cytokines and scaffolds in order to improve their effectiveness. The most common scaffolds used are hyaluronic acid and fibrin gel. PRP has been applied as a nutrient fluid containing chondrogenic and osteogenic growth factors (e.g., TGF- $\beta$ and PDGF) to support the survival and differentiation of transplanted MSCs [23,24].

\section{Who are the RSU Patients? A focus on OA and hernia repair}

Joint and back pain is often the result of degenerative $O A$ and is one of the most frequent causes of patient visits for assessment. Degenerative Osteoarthritis $(O A)$ is the most common chronic musculoskeletal disorder. Whilst $O A$ can affect all joints, the knee, spine, hip, hands, feet, and shoulder are most frequently involved. Chronic pain leading to disability is the most common direct consequence. $O A$ is the single most common cause of disability in older adults [2].

$O A$ risk factors include obesity, sedentary lifestyle, chronic postural defects, bone density, occupational injury, trauma, and genetic predisposition.

The 2010 Global Burden of Disease Study estimated that $10 \%$ to $15 \%$ of all adults aged over 60 had some degree of symptomatic $O A$, with prevalence higher among females than males [25,26].

Most of the current medical treatments are palliative and work on pain management and symptom control. In advanced $O A$, total knee and hip replacement are the current standards to alleviate pain and improve function. However, the risks associated with the operation 
and the finite life span of joint prosthesis exclude this option for a significant group of patients.

Joint replacement cannot often be offered to frail elderly patients (ASA III-IV) due to high risks of complications and increased mortality. Likewise, surgeons are reluctant to offer to younger patient's joint replacement due to the limited life span of prosthesis and chronic postural imbalance induced by the new joint [2]. The result is the increasing consumption of chronic medications such as NSAIDs and opioids in socalled pain management. This palliation has a severe systemic backlash on patient care and health systems due to the complications related to chronic NSAIDs and opioids intake and addiction [2].

Dilemmas can arise when we seek to offer patients the greatest benefits along with minimal risks. The basic medical principles primum non nocere, secundum cavere, tertium sanare (first do not harm, second be careful, third cure) should always guide and inspire medical practice [2].

The latest knowledge about the biological processes involved in the early $\boldsymbol{O A}$, has given general awareness that this disease can be curbed and reversed if addressed at the early stage [2].

Early $O A$ is defined by recurrent joint pain together with no or minimal changes detectable by X-ray imaging. Only MRI and USS can identify early markers of early OA in the synovial membrane and some superficial cartilage erosions [27-29].

These methods and criteria enable early identification of joint disease and this facilitates early therapeutic approaches to halt and reverse the normal progress of the disease $[2,30]$.

The development of regenerative medicine procedures has facilitated a major change in early $O A$ treatments as well as in advanced $O A$. These therapies provide persistent analgesia through tissue repair for frail elderly patients as well as younger patients who are currently offered simple palliation [2].

In addition, regenerative surgery minimally invasive procedures are non-toxic, very low risk and have minimal adverse side effects.

Inguinal hernia repair is the most frequently performed operation in General Surgery. Over 20 million hernia repair procedures per year are performed worldwide [8].

Complications including chronic inguinal pain (12\%) and recurrence rate $(11 \%)$ have significant negative impact on surgical results. The 4 main impacting factors affecting hernia repair results are: 1) mesh material and design; 2) mesh fixation; 3) tissue healing and regeneration and 4) the surgical technique [8].

PRF-hernia repair is a new physiologic and genuinely "tensionfree technique" that follows the sound principles of both regenerative and conventional surgery. $L-P R F$ mesh fixation removes the need for stitches, the usual cause of "nerve entrapment syndrome" due to the snaring by stitches of inguinal nerves. Further, the $P R F$ antiinflammatory and tissue regeneration properties both speed and 'streamline' the process of mesh integration.

The surgical technique, mesh material choice, biological method of fixation and the regenerative properties of $P R F$ work synergistically to minimize wound site inflammation and assist correct integration of the mesh. These result in postoperative advantages. The short-term benefits are less pain and shorter time of recovery $[8,9]$.

The mid-long-term benefits are lower incidence of chronic pain due to a correct process of wound healing and minimizing the risk of nerve entrapment $[8,9]$.
Better integration and minimization of scar tissue may lead to a stronger repair and associated reduction in recurrence. This can be confirmed by long-term studies conducted by the research asset.

\section{Other areas of expertise in the RSU}

As reported in the $R S U$ model, patients affected by chronic wound and skin ulcers such as diabetic foot ulcers (DFU), pressure ulcers (PU); venous leg ulcers (VLUs) have been treated. Significant benefit resulted from treatments of debridement together with the application of PRF membrane enriched with HSPs (supercharged-PRF).

$P P R P$ and ASCs aesthetic regenerative fillers have been used to treat skin defects and to minimize wrinkles. $P R P$ in combination with HSPs has been applied for hair regrowth and to aid transplantation.

These procedures involve different specialists and health professionals working cooperatively in the RSU multidisciplinary model.

\section{Establishing an alliance between doctor and patient}

The relationship between doctor and patient should be based on ethics, professionalism, competence, trust, honesty and especially on empathic communication.

The current process to gain the informed consent of patients has focused on medico-legal aspects. In daily practice, a doctor is mostly dedicated to defending his/her-self from potential complaints. The consent goal is to get permission to deliver medical treatments and the patient's acceptance of complications; these are usually stated in a long list. In the end, doctors effectively request the patient's permission to accept treatments and possible adverse events.

This is quite different to a relationship based on awareness and trust. In our model, the consent process is an essential step to establish a relation of partnership with the patient. Open and honest communication will satisfy patients that regenerative surgery procedures are the safest of the current medical options and effective enough to attempt them before considering major invasive procedures. This is in accordance with the first basic medical principle first do no harm. Doctors must mention that failure of treatment is a possible outcome. It is also proper to mention that the primary ethical aim is to attempt to remove suffering by improving patient symptoms with the least invasive and least harmful treatment that is currently available and has strong indications of efficacy.

The overall goal is to establish an alliance between the doctor and patient to pursue the common goal of best result and patient satisfaction.

\section{RSU ambulatory care and day-surgery settings}

Most of the $R S$ procedures can be delivered in ambulatory care settings. This model has been applied in Villa Aurora HospitalRegenerative Surgery Unit- Foligno (Italy). The essential parts are summarized below.

A dedicated laboratory area hosts centrifuges, fridge, oven-dryer and homogenizer where the patient's blood or adipose tissue can be sterilely manipulated and prepared.

Adjacent clinical rooms are arranged to deliver $R S$ procedures. These need to be set as a surgical ambulatory care facility, each tailored for the specialist services to be provided: e.g. orthopedics, plastic surgery, dental service etc. 
USs with a linear probe should be always available for $O A$ treatments.

Main areas in the RSU ambulatory care settings:

- Reception and waiting room

- Blood Sample collection

- RS laboratory to prepare individual medication

- RS ambulatory care treatments

- Recovery, refreshment, and discharge

Procedures that can be delivered by ambulatory services are mainly $P R P / P R F$-derivatives and monocytes. These treatments provide valid therapeutic options in OA patients, implantology and plastic/aesthetic procedures.

To elicit best results in $O A$, particular care must be taken over the method of $P R P$ production and application and the accurate positioning of the bio-stimulator in the affected joints. Centrifuge characteristics, centrifugation speed/ time, manipulation of the resulting product(s) and positioning in the joint tissue can all change the effectiveness and therefore the results of $P R P s$ procedures [31].

We have used autologous adipose stem cells (ASCs) procedures for $O A$ in various combinations with $P R P$ and scaffolds to improve clinical results. ASCs procedures are generally delivered under general anesthetic in a day surgery facility. PRF-inguinal hernia repair is a day surgery new technique delivered in theatre setting. The centrifuge to produce the $L-P R F$ used to fix the mesh is based in theatre.

\section{Our research and innovative procedures}

Stimulation of resident MSCs. Most joint tissue regeneration treatments have targeted cartilage tissue. However, literature on the physiopathology of early $O A$ has shown the essential role played by the synovial membrane and synovial fluid to trigger the disease together with articular cartilage modifications [2]. Joint pain is firstly initiated by changes to non-cartilaginous components such as the joint capsule, synovial membrane, tendons and ligaments where there is a significant innervation [32,33].

Since 2012 the author has published work on procedures whose core effect is stimulation of joint resident MSCs [34,35]. A study mentioned in the work aimed to show long-term symptom control through the recovery of local cell homeostasis and tissue repair. The study examined an individual medication comprised of polydeoxyribonucleotides $(P D R N)$, a specific set of Heat Shock Proteins (HSPs) and a glycerol scaffold. The HSPs were derived from the patient's blood through a process of thermic stress.

This product named $\mathrm{Gel}$ repair ${ }^{\circ}(G R)$ has shown to be a biological activator able to stimulate the resident MSCs located in the capsule and synovial tissue.

An observational clinical trial with 3 years follow-up showed a persistent improvement of symptoms together with a radiologic recovery of joints affected by degenerative Osteoarthritis. Lower pain level and increased joint mobility were observed in almost $80 \%$ treated patients and imaging often showed a downgrading of the degenerative $O A[34,35]$. Besides cartilage and bone regeneration, Gel Repair ${ }^{\circledR}$ (in some preliminary experiences) has shown wound healing properties on chronic skin ulcers that did not respond to traditional treatments. Following the same biological principles, we have been using thermic stressed $L-P R F$ membranes to produce a pool of HSPs. The process aims to activate SM-MSCs (Synovial Membrane-MSCs) in OA. The resulting product, termed 'supercharged $L-P R F$ ' when used in a procedure, supplements the stimulation of SM-MSCs with a pool of HSPs that are slowly released by the treated membranes when applied on the surface of capsule-bursa tissues.

Omentum adipose stem cells (OASCs) transplantation has been successfully used for muscle regeneration, for degenerative osteoarthritis and to prevent colorectal anastomotic leak [17].

\section{Scientific activity}

$R S U$ implements data collection and statistical analysis to measure the quality of services and their performance. Data and results will eventually be published in scientific journals.

This approach improves the quality of health care in any sector of $R S U$ activities. The clinical activity is set by default as a longitudinal, observational, or comparative study to constantly deliver research results on the ground. This is the basis for CQI/TQM best practice in clinical settings [36]. This measurable systematic approach generates quality, high performance, and value. The RSU-members website promotes regenerative surgery information and updating, interactive communications, e-learning, and training. The website will outline and enable members to explore options for a scientific society/association model.

One option has a board of scientists, academics, experts in biology, immunology, regenerative surgery, longevity, and regulatory professionals. The association may elect to establish partnerships with universities and other institutions to promote educational events and research.

\section{Other services associated with the RSU}

In orthopedics, $R S U$ works in a team with physiotherapists and advises long term swimming-pool-physio to improve the quality and duration of results. Furthermore, OA patients may be obese and /or frail and/or with multiple chronic comorbidities. Therefore, we offer specialistic consultations and treatments to tackle these problems and prevent $O A$ recurrence.

\section{Health tourism}

Health or Medical tourism refers to people travelling abroad to obtain medical treatments or health care services. Historically, patients and people have always travelled from developing countries to developed countries to receive medical treatments unavailable in their countries [37].

However, in recent years, health tourism has become a two-way flow. Social and economic motivations have pushed people from developed countries to seek treatments in developing countries that promise high performance at lower prices. Moreover, tourism boards of some countries offer medical care together with touristic/ cultural excursions and entertainment. There are significant differences between the medical regulatory agencies in various countries (FDA; EMA etc.). As a consequence, a drug or a procedure may be approved in some countries and not in others. Therefore, another reason for Medical tourism is to receive medical care unavailable or non-licensed in the home country.

Medical tourism most often is for plastic and cosmetic surgeries, dental and fertility procedures [38]. More recently, 'longevity' therapies involving stem cells and associated treatments are rapidly 
expanding in several medical fields. Again, the core aim is regeneration of dysfunctional cells, tissues, organs and structures. Medical Tourism offers ought to warrant safety, effectiveness, quality of services, high performance, assessment should be handled by an independent quality commission.

Our RSU located at Villa Aurora Hospital- Foligno (Italy) is fully accredited by the National Health System (SSN) and rated by external commissioners. Foligno is a medieval town close to Perugia in the central heart of Italy (Umbria) which offers beauty and peace in enchanting scenarios together with art and culture.

\section{Acknowledgments}

The author thanks Mr Thomas Richard Swift for the cultural discussions and the English revision of the manuscript.

\section{References}

1. Zhu Y, Yuan M, Meng HY, Wang AY, Guo QY, et al. (2013) Basic science and clinical application of platelet -rich plasma for cartilage defects and osteoarthritis: a review. Osteoarthritis Cartilage 21:1627-1637. [Crossref]

2. Di Nicola V (2020) Degenerative Osteoarthritis a reversible chronic disease. Regen Ther 15: 149-160.

3. Laudy AB, Bakker EW, Rekers M, Moen MH (2015) Efficacy of platelet -rich plasma injections in osteoarthritis of the knee: a sistematic review and meta-analysis. $\mathrm{Br} J$ Sports Med 49: 657-672.

4. Mishra A, Tummala P, King A, Lee B, Kraus M, et al. (2009) Buffered PlateletRich Plasma Enhances Mesenchymal Stem Cell Proliferation and Chondrogenic Differentiation. Tissue Eng Part C Methods 15: 431-435. [Crossref]

5. Krüger J, Hondke S, Endres M, Pruss A, Siclari A, et al. (2011) Human platelet-rich plasma stimulates migration and chondrogenic differentiation of human subchondral progenitor cells. J Orthop Res 30: 845-852. [Crossref]

6. Mifune Y, Matsumoto T, Takayama K, Ota S, Li H, et al. (2013) The effect of plateletrich plasma on the regenerative therapy of muscle derived stem cells for articular cartilage repair. Osteoarthritis Cartilage 21: 175-185. [Crossref]

7. Bendinelli P, Matteucci E, Dogliotti G, Corsi M, Banfi G, et al. (2010) Molecular basis of anti-inflammatory action of platelet-rich plasma on human chondrocytes: Mechanisms of NF-אB inhibition via HGF. J Cell Physiol 225: 757-766. [Crossref]

8. Di Nicola V (2020) Regenerative Surgery in Open Mesh Repair for Inguinal Hernia. J Regen Med 6: 307.

9. Di Nicola V (2020) Regenerative Surgery for Inguinal Hernia Repair. Clin Res Trials 6: $1-8$.

10. Caplan AI, Correa D (2011) The MSCs: an injury drugstore. Cell Stem Cell 9: 11-15. [Crossref]

11. Gao F, Chiu SM, Motan DA, Zhang Z, Chen L, et al. (2016) Mesenchymal stem cells and immunomodulation: current status and future prospects. Cell Death Dis 7: e2062. [Crossref]

12. Najar M, Raicevic G, Crompot E, Fayyad-Kazan H, Bron D, et al. (2016) The Immunomodulatory Potential of Mesenchymal Stromal Cells: A Story of a Regulatory Network. J Immunother 39: 45-59. [Crossref]

13. Najar M, Raicevic G, Fayyad-Kazan H, De Bruyn C, Bron D, et al. (2015) Bone Marrow Mesenchymal Stromal Cells Induce Proliferative, Cytokinic and Molecular Changes During the T Cell Response: The Importance of the IL-10/CD210 Axis. Stem Cell Rev Rep 11: 442-452. [Crossref]

14. Caplan AI (2019) Mesenchymal Stem Cells in Regenerative Medicine. Principle of Regenerative Medicine Ch 15. (3 ${ }^{\text {rd }}$ edn), Elsevier Inc.

15. Rothrauff BJ, Pirosa A, Lin H, Sohn J, Langhans MT, et al. (2019) Stem Cells for Musculoskeletal Diseases. Principle of Regenerative Medicine Ch 54( $3^{\text {rd }}$ edn), Elsevier Inc.

16. Javazon EH, Begges Kj, Flake AW (2004) Mesenchymal stem cells: paradoxes of passaging. Exp Hematol 32: 414-425;18. [Crossef]

17. Di Nicola V (2019) Omentum a powerful biological source in regenerative surgery. Regen Ther 11: 182-191.
18. Maumus M, Pers YM, Ruiz M, Jorgensen C, Noël D (2018) Mesenchymal stem cells and regenerative medicine: future perspectives in osteoarthritis. Med Sci (Paris) 34: 1092-1099. [Crossref]

19. Spees JL, Olson SD, Whitney MJ, Prockop DJ (2006) Mitochondrial transfer between cells can rescue aerobic respiration. Proc Natl Acad Sci U S A 103: 1283-1288. [Crossref]

20. Pittenger M (2009) Sleuthing the source of regeneration by MSCs. Cell Stem Cell 5 8-10. [Crossref]

21. Iwashima S, Ozaki T, Maruyama S, Saka Y, Kobori M, et al. (2009) Novel culture system of mesenchymal stromal cells from human subcutaneous adipose tissue. Stem Cells Dev 18: 533-543. [Crossref]

22. Riordan NH, Ichim TE, Min WP, Wang H, Solano F, et al. (2009) Non-expanded adipose stromal vascular fraction cell therapy for multiple sclerosis. J Transl Med 7: 29. [Crossref]

23. Freitag J, Bates D, Boyd R, Shah K, Barnard A, et al. (2016) Mesenchymal stem cell therapy in the treatment of osteoarthritis: reparative pathways, safety and efficacy - a review. BMC Musculoskelet Disord 17: 230. [Crossref]

24. Koh YG, Jo SB, Kwon OR, Suh DS, Lee SW, et al. (2013) Mesenchymal stem cell injections improve symptoms of knee osteoarthritis. Arthroscopy 29: 748-755. [Crossref]

25. WHO, Department of Chronic Diseases and Health Promotion. Available from: http:/ www.who.int/chp/topics/rheumatic/en

26. United Nations. World Population to 2300. Available from: https://www.un.org/en/ development/desa/population/publications/pdf/trends/WorldPop2300final.pdf

27. Chiba D, Nakamura T, Fu FH (2019) Early osteoarthritis definition, pathogenesis, diagnosis, management and prevention: management. AOJ: 4.

28. Di Nicola V, Di Pietrantonio M (2016) MRI in Degenerative Joint Disease (DJD): A Proposal for Imaging Standardization in Regenerative Medicine. SM Musculoskelet Disord 1: 1006.

29. Kaeley G.S, Bakewell C, Deodhar A (2020) The importance of ultrasound in identifying and differentiating patients with early inflammatory arthritis: a narrative review. Arthritis Res Ther: 22

30. Luyten FP, Denti M, Filardo G, Kon E, Engebretsen L (2012) Definition and classification of early osteoarthritis of the knee. Knee Surg Sports Traumatol Arthrosc 20: 401-406. [Crossref]

31. Doan Ehrenfest DM, Pinto NR, Pereda A, Jiménez P, Corso MD, et al. (2018) Impact of the centrifuge characteristics and centrifugation protocols on the cells, growth factors, and fibrin architecture of a leukocyte- and platelet-rich fibrin (L- PRF) clot and membrane. Platelets. 29: 171-184. [Crossref]

32. Mashaghi A, Marmalidou A, Tehrani M, Grace PM, Pothoulakis C, et al. (2016) Neuropeptide substance P and the immune response. Cell Mol Life Sci 73: 4249-4264. [Crossref]

33. Mora JC, Przkora R, Cruz-Almeida Y (2018) Knee osteoarthritis: pathophysiology and current treatment modalities. J Pain Res 11: 2189-2196. [Crossref]

34. Di Nicola V, Di Nicola R (2012) Self-repair in degenerative joint disease. Curr Aging Sci 5: 273-287. [Crossref]

35. Di Nicola V, Pierpaoli W (2013) Biological baseline of joint self-repair procedures Curr Aging Sci 6: 206-214. [Crossref]

36. Shortell SM, O’Brien JL, Carman JM, Foster RW, Hughes EF, et al. (1995) Assessing the impact of continuous quality improvement/total quality management: concept versus implementation. Health Serv Res 30: 377-401. [Crossref]

37. Horowitz MD, Rosensweig JA, Jones CA (2007) Medical Tourism: Globalization of the Healthcare Marketplace. Med Gen Med 9: 33. [Crossref]

38. Medical tourism--health care in the global economy (PDF). Physician Exec. Retrieved 16 September 2012.

Copyright: (C2020 Valerio Di Nicola. This is an open-access article distributed under the terms of the Creative Commons Attribution License, which permits unrestricted use, distribution, and reproduction in any medium, provided the original author and source are credited. 